\title{
Intra-Body Temperature Monitoring using a Biofeedback Solution
}

\author{
João M. L. P. Caldeira ${ }^{1,2}$, José A. F. Moutinho ${ }^{3}$, Binod Vaidya ${ }^{4}$, Pascal Lorenz ${ }^{5}$, and Joel J. P. C. Rodrigues ${ }^{1,4}$ \\ ${ }^{1}$ Department of Informatics, University of Beira Interior, Covilhã, Portugal \\ ${ }^{2}$ EST, Polytechnic Institute of Castelo Branco, Castelo Branco, Portugal \\ ${ }^{3}$ Health Sciences Faculty, University of Beira Interior, Covilhã, Portugal \\ ${ }^{4}$ Instituto de Telecomunicações, Covilhã, Portugal \\ ${ }^{5}$ IUT, University of Haute Alsace, Colmar, France \\ jcaldeira@est.ipcb.pt,jafmoutinho@fcsaude.ubi.pt,bnvaidya@co.it.pt,lorenz@ieee.org,joeljr@ieee.org
}

\begin{abstract}
Body Area Sensor can enable healthcare monitoring in an unobtrusive way. Medical and healthcare research has been striving to find relationships between core body temperature at female genitals and certain health conditions, such as ovulation period. This paper presents a solution for intra-body temperature monitoring based on a new intra-body sensor, communication and desktop application tool. This new biosensor provides data collection that may be used to study the relation between temperature variations and women health conditions, such as, ovulation period (for both natural contraception and in vitro fertilization purposes) among others. The motivation for this work focuses on the creation of this eHealth solution that will fill the gap we realize in medical technology. The proposal was tested and validated by a medical team and it was concluded that this new biosensor performs perfectly.
\end{abstract}

Keywords - Biosensor; Wireless sensor network; Biofeedback; e-Health; Temperature Monitoring.

\section{INTRODUCTION}

Body area sensor networks (BASNs) are emerging technologies that promise novel uses in healthcare and other medical applications. Each BASN consists of multiple interconnected nodes on, near, or within a human body, which together provide sensing, processing, and communication capabilities.

Recent publications [1-3] report continuous evolution and progress of new biosensors for healthcare and biofeedback. These sensors became indispensable in the daily routine of medical staff where they have the capability for helping medical procedures and healthcare. Nowadays, biosensor systems are powerful available instruments in diagnosis, controlling, monitoring and prevention of some diseases [4, 5]. In some cases, they also became an essential instrument for heal support [6-8].

The evolution of these biosensors offers a new range of the infinity possibilities for applications they can provide. The miniaturized size of these nodes turns these systems more easy to use, in a comfortable way. They can access to inside-human body places that were difficult to reach and non comfortable for patients, using traditional methods [9]. Advantages of these systems and the great interest of medical community turn this research area as an important topic.

The accurate measure of core body temperature of a human being is a physiologic indicator that is very important in numerous medical applications. Several ideal sites such as pulmonary artery, bladder have been identified for monitoring core body temperature. Placing a sensor in some of these body parts is considered invasive.

To the best of our knowledge, none of the existing biosensor could be capable to measure intra-vaginal temperature continuously. Therefore, the main goal of this work is proposal, creation, test and validation of a novel intra-body sensor for vaginal temperature acquisition and monitoring. The motivation for this work focuses on the creation of this new biosensor that will fill the gap we realize in medical technology.

This work was conducted jointly with a medical team from Health Sciences Faculty of University of Beira Interior, Covilhã, Portugal. This new biosensor allows the execution of exploratory studies to increase the knowledge of female intra-vaginal physiological behavior. To perform this study, medical team wants to monitor and analyze the intra-vaginal temperature during the female menstrual cycle. Furthermore, they will use this new system in the following applications: preterm labor prevention, detection of pregnancy contractions, anticipation and monitoring of the ovulation period (for both natural contraception and in vitro fertilization purposes), effectiveness of some gynecology therapeutics, and supporting the discovery of new possible contraception methods. These system applications will be conducted taking into account previous medical studies where this physiological parameter (the temperature) is correlated with several human phenomena [10-12], including female fertility issues [13]. This confirms the importance of the contribution presented in this paper.

The remainder of the paper is structured as follow. Section 2 presents some related works, while Section 3 describes the 
new sensor architecture. Section 4 includes the wireless communication between the biosensor and the application software to collect and treatment of collected data. Section 5 presents the biosensor validation and includes several results performed the proposed solution. Finally, Section 6 concludes the paper and points further research directions.

\section{RELATED WORKS}

Medical and healthcare research has been striving to find relationships between core body temperature at female genitals and certain health conditions, such as ovulation period. A study presents some conclusions on the correlation between covert attention and basal temperature changes during the menstrual cycle phase on 22 adult females proves the importance of basal (intra-vaginal) temperature [10]. In this study, traditional way was used for temperature measurement. However, automatic measurements and analysis of intra-vaginal temperature readings in an unobtrusive and efficient way are desirable.

Another study uses a radio pill created for astronaut use, to access internal body temperature on athletes, and take measures to cool them down, avoiding excessive fatigue [14]. However, such pill-based solution introduces issues on pill elimination, and the biosensor cannot be reused again.

Other medical studies, developing integrated systems to acquire and monitor physiological parameters, including body temperature $[7,15]$. These systems only measure the skin temperature. From [11] we can see that skin temperature cannot reflect the basal body temperature as it varies depending on the ambient temperature. The AMON research team included a temperature sensor on their wearable system (AMON) [7] to study a possible correlation between the temperature readings taken at skin by the sensor and the core body temperature. They concluded that skin temperature could be influenced by the environment conditions. Therefore, they could not show any correlation between skin temperature and core body temperature. DuoFertility project [15] created a system to predict women fertile period. This system bases its prediction on the measurement of skin temperature. During fertile period, the variation of women core body temperature occurs around 10-14 days of menstrual cycle. It only changes about 0.5 degree Celsius [13]. Thus, trying to get core body temperature by measuring skin temperature could lead to wrong interpretations and conclusions.

In paper [16], the authors presented a system using UHF radio telemetry to measure the vaginal temperature and monitor the temperature. While in paper [17], the author proposed a highly accurate system in which a capsule shaped sensor measures the central body temperature. This sensor can be ingested or inserted rectally such that it will transmit core body temperature continuously.

Next sections present, in detail, the construction of the new intra-vaginal temperature biosensor, and the corresponding application and communication system. This system deploys results on a personal computer for monitoring and further analysis.

\section{INTRA-BODY BIOSENSOR}

Sensing is fundamental to all sensor networks, and its quality depends on many factors such as size, material used.

Physiological sensors measure core body temperature, ambulatory blood pressure, blood oxygen etc. As the accurate measure of core body temperature is highly preferred in numerous medical applications, intra-body biosensor is required. The main challenge is the construction of a novel intra-body biosensor for intra-vaginal temperature monitoring.

Furthermore, the intra-body biosensor must consider the sensitivity of the body area, critical for the comfort of the user on a daily basis.

This proposal falls in the conception of a novel biosensor device to measure intra-vaginal temperatures and continuously gather their measurements for further analyses purpose. To access and analyze all data collected by this intra-body biosensor, a new application was also developed.

The novel biosensor system was created with the help of SHIMMER platform (Sensing Health with Intelligence Modularity, Mobility and Experimental Reusability), which is a wireless sensor platform designed by Intel and can be used as the central processor unit of the biosensor network. This platform has a Texas Instruments MSP430 CPU $(8 \mathrm{MHz})$, a class 2 Bluetooth radio communication, an IEEE 802.15.4 Chipcon wireless transceiver $(2.4 \mathrm{GHz})$, a 3 -Axis Freescale accelerometer, a MicroSD slot for up to 2Gbytes, an integrated Li-Ion battery management and some extensions to append new features and functionalities mounted in a very small form factor $(2 \times 4.5 \mathrm{~cm})$ not larger than a thumb size. This platform is an ideal hardware for this work due to its small size, large data storage capacity and communication features.

However, there is no temperature sensor installed on SHIMMER platform. In order to get temperature readings, a temperature sensor must be integrated into SHIMMER. Thus, the MA100 thermistor from GE Industrial Sensing is used for this solution. Its sensitivity ranges from 0 to 50 degree Celsius, size is $0.762 \times 9.52 \mathrm{~mm}$, and is created for 
biomedical applications. MA100 is connected to the SHIMMER with a flexible cable.

Due to bulky size, SHIMMER is not possible to place it inside the female cervix. Therefore, only MA100 thermistor is introduced inside the vagina and sends measurements (in voltage) to SHIMMER, using the flexible cable. The SHIMMER unit stays outside of the women body and could be placed anywhere if the cable is long enough $(\sim 80$ centimeters). Because the cable is very flexible and difficult to handle inside the female cervix, we created a tampon-like enclosure to enclose the biosensor. This solution enables such that women can easily introduce the tampon-like enclosure with temperature sensor inside reproductive system because it can be used as ordinary tampons.

Figure 1 presents the proposed sensor architecture. The temperature sensor (MA100) is placed inside the female cervix, while the processor unit (SHIMMER) remains outside. Bluetooth performs the communication between this device and the computer.

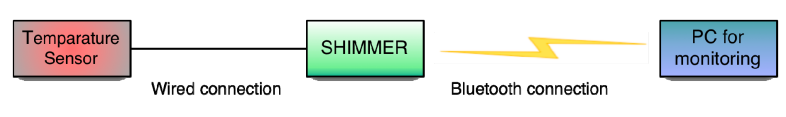

Figure 1. System architecture.

To ensure the acquisition and storage of the temperature reading values, a SHIMMER firmware was developed. This software, running in continuous mode, is waiting for personal computer commands over a Bluetooth connection. Once a command is arrived, SHIMMER analyzes it and proceeds in accordance. The available commands in SHIMMER firmware are the following: start collecting the temperature values in microSD, stop collecting, turn on a red led (for debugging purposes), programming the interval between temperature readings and send all data recorded in microSD to the personal computer application.

We can get convincing results only if we can identify the correspondence between the measured temperature and the exact time it was acquired. SHIMMER has a local time clock starting on the startup time however it does not have a global time clock. In order to provide a global time clock on SHIMMER, we have devised such that when computer performs a start collection command, it also sends its time and date (assuming that computer clock is synchronized with global time clock). This information is used on SHIMMER, regarding is local time, to calculate the global time clock associated with each measure.

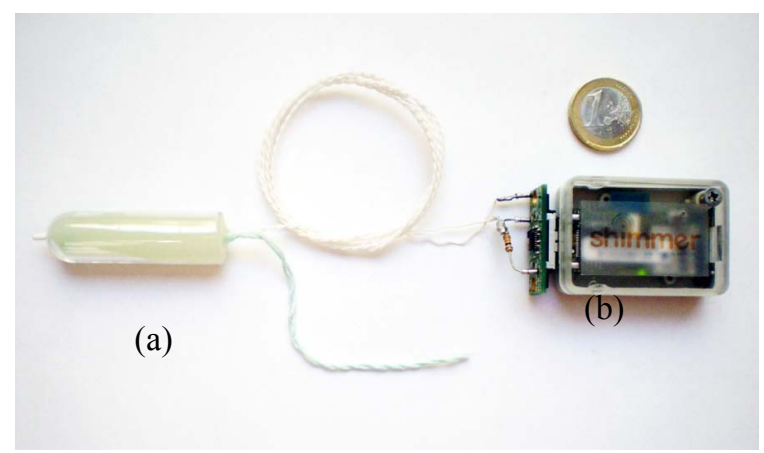

Figure 2. Intra-vaginal sensor. (a) MA100 thermistor with enclosure; (b) SHIMMER platform.

The proposed biosensor in this paper is presented in Figure 2. Figure 2(a) shows the MA100 thermistor in its enclosure, which is placed inside the female cervix. It can be seen that tampon-like enclosure with MA100 thermistor is made such that it is rather straightforward to use. The SHIMMER platform and the external extension where MA100 is connected may be seen in Figure 2 (b).

\section{COMMUNICATION AND APPLICATION TOOL}

\section{A. Communication}

Although SHIMMER is a powerful biosensor platform, with all the above-mentioned described features, it has very limited resources in terms of computation and is very dependable from a power battery with no long lifetime. In order to ensure increase of the battery lifetime, we only gather temperature readings from SHIMMER, instead of collecting and processing them. Temperature readings are transmitted to a computer and collected by an application for further analysis and processing.

Bluetooth performs the communication between SHIMMER and a personal computer. Wireless communications seems to be more realistic than other wired alternatives, taking into account patients comfort and operation simplicity by medical staff.

Like any BASNs, it is unique in that it attempts to restrict the communication radius to the body's periphery. Limiting transmission range reduces a node's power consumption, decreases interference, and helps maintain privacy.

As expected, the connection between the biosensor and computer is only available if the sensor is in Bluetooth's connection range to the computer. Because of that, we cannot have the effective monitoring of the temperature values if the sensor is out of range. Therefore, in case of 
communication failure between SHIMMER and the personal computer, SHIMMER only collects temperature and saves temperature readings on its local microSD such that it can transmit them when the Bluetooth connection is active. This procedure prevents unnecessary use of power to perform the communication and increases battery's lifetime. Figure 3 shows a diagram of Bluetooth data transmission presenting the procedure performed in case of existence of an active Bluetooth connection, or not.

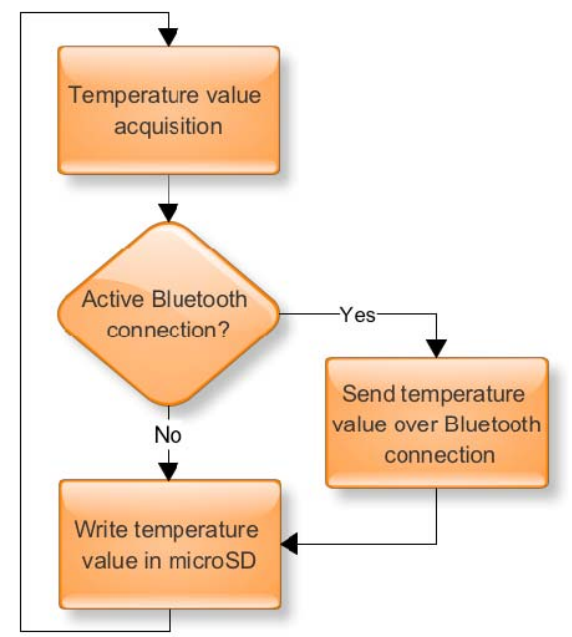

Figure 3. Diagram of Bluetooth data transmission, existing an active connection or not.

\section{B. Application Software}

An application to collect, process, analyzes and visualize in a personal computer the reading measures performed by the intra-vaginal sensor was created. To achieve intra-vaginal sensor temperature values, both real-time and off-line operation modes are available.

In the real-time mode, measured values can be achieved when SHIMMER is Bluetooth connected with the computer. In this case, the application software shows the real-time temperature values measured by SHIMMER. Simultaneously, all the temperature measures are send to the connected computer, via Bluetooth, and written on the microSD card of SHIMMER platform.

In the off-line mode, the application software can collect all the long-term temperature readings stored in the microSD card. The application software can retrieve all the stored data on the SHIMMER's microSD card. These data may be both visualized and saved in a text file for further use, if needed.
Figure 4 shows a screenshot of the application software to program, operate, and control the intra-vaginal temperature biosensor. It performs all the following system features:

- Define the time interval between each temperature reading (e.g., $1 \mathrm{sec}, 30 \mathrm{sec}, 1 \mathrm{~min}, 5 \mathrm{~min}$ and $10 \mathrm{~min}$ ).

- $\quad$ Start the acquisition of SHIMMER's temperature readings. SHIMMER start collecting the reading values and save them on the microSD card.

- $\quad$ Stop data acquisition.

- Collect temperature readings stored on SHIMMER's microSD. Application import data from the microSD and also may save them in a text file for persistent storage.

- Visualize the temperature reading values. Using Switch Led button, user may choose between realtime or off-line mode to observe the measured values.

As the application interacts directly with SHIMMER, these features are only available if Bluetooth connection is available between computer and SHIMMER.

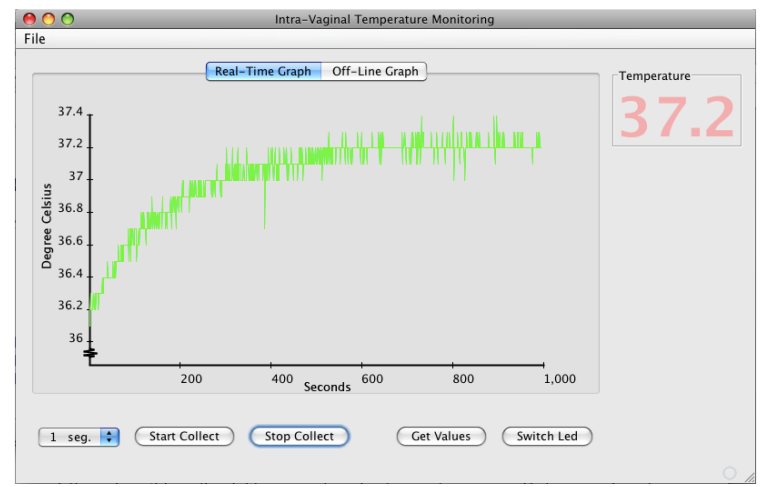

Figure 4. Personal computer application for intra-vaginal temperature monitoring.

As may be seen in Figure 4, temperature values are presented using a graphical representation. Thus, it is easy to visually identify values outside the normal pattern. These values can lead to sign a range of conditions on female reproductive system (e.g., pregnancy contractions, ovulation period, best fertilization period, etc.). Next, a medical research will carried out with the execution of very important studies to be applied in different kind of gynecology issues, such as, the preterm labor prevention, detection of pregnancy contractions, anticipation and monitoring of the ovulation period (used either as a natural contraception method or, at the opposite, as a estimation of the best fertilization period), effectiveness of some gynecology therapeutics, and supporting the discovery of new possible contraception methods. 


\section{SOLUTION VALIDATION}

Comfort, usability and, mainly, accuracy were the goals that were followed in the construction of the intra-vaginal sensor. These targets have to be tested and validated in a real environment. Thus, medical team conducted several tests to perform the solution validation.

The biofeedback solution for intra-vaginal temperature monitoring was tested in 12 hospitalized women. Every woman was monitored for about 60 minutes with temperature readings per second. Simultaneously, women body temperatures under the arm and under the tongue, was measured using a trivial digital thermometer. After analyzing all the temperature data, medical team validated and certified the accuracy of intra-vaginal temperature readings. After test finishing, each woman was questioned about the possible discomfort caused by the use of the intravaginal sensor. None of them showed any issue related to the use of this biosensor.

The sensor was also tested in normal day life with 8 women. These tests was preformed in periods of about $2 \sim 3$ hours of the day. The results were compared to patterns previously defined with traditional thermometers measures in same places above (under the arm and under the tongue). The medical team confirmed the validation of the results.

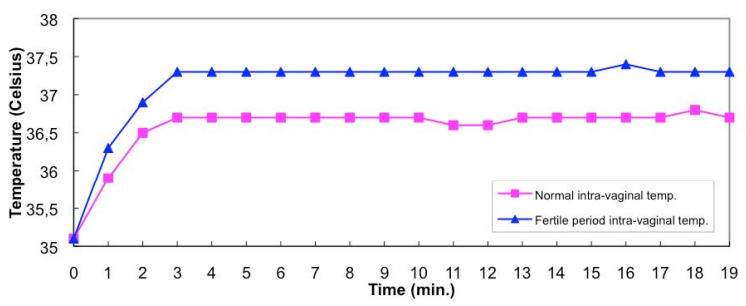

Figure 5. Sample of intra-vaginal temperatures measurements in two different days for the same woman in normal day life.

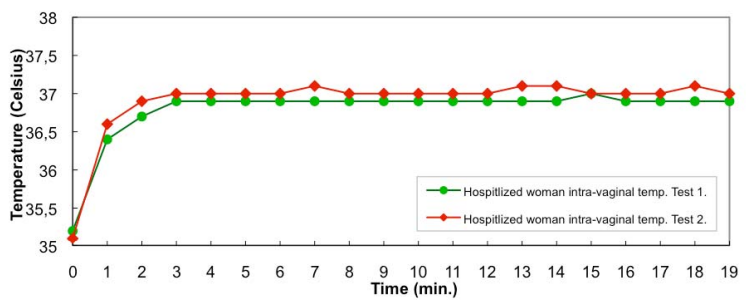

Figure 6. Sample of intra-vaginal temperatures measurements in two hospitalized women.

Figures 5 and 6 present sample of temperature results obtained during tests. In Figure 5, one may be seen temperature values performed by the intra-vaginal sensor for the same woman in tow different days. This graph could be divided in two segments for both curves. First segment, between minute 0 and 2, represents the heating of the sensor to reach ambient temperature (sensor response). The second segment, beyond the 3 rd minute, represents the real temperature readings inside woman vagina. Differences between both temperature readings, beyond the 2nd minute, correspond to the normal intra-vaginal temperature of this woman and her temperature on a fertile period (ovulation period). As expected, intra-vaginal temperature changes according to some female body situations. Monitoring this parameter it could help in medical detection of these situations, such as, the preterm labor prevention, detection of pregnancy contractions, anticipation and monitoring of the ovulation period (used either as a natural contraception method or, at the opposite, as a estimation of the best fertilization period), effectiveness of some gynecology therapeutics, and supporting the discovery of new possible contraception methods.

Figure 6 presents a sample with results of two tests performed in two of the twelve hospitalized women evaluated. In this graph, the two above-mentioned segments could also be distinguished. From 0 to 2 nd minute, it is the response of the sensor and beyond the 3rd minute (after its stabilization), it represents the real temperature values inside women vagina. As may be seen, beyond 3rd minute, differences between both curves means (and confirm) that each woman have her own intra-vaginal temperature. By this reason, individual patterns may have to be established for each woman.

These results represent a great success and encourage medical team for more patients' data collection, using this new biosensor sensor, trying to establish patterns of intravaginal temperature behavior. Further, these patterns will be used to understand the relationship between intra-vaginal temperature variations and some reproductive system behaviors, as well as the comparison between patterns and new data collections.

\section{CONCLUSION AND FURTHER WORK}

This paper proposed a new biofeedback solution for intrabody temperature monitoring by not only creating a new intra-body biosensor for collecting temperature readings at the female genitals but also by developing appropriate application software for processing, analyzing, and visualization of the collected data. This system was tested and validated by medical team, both in day life and hospitalized women, with success. The accuracy of the results was also confirmed. Next, medical team will conduct several studies with this system in order to study and try to recognize some phenomena related with reproductive 
system behavior. These studies may contribute to the preterm labor prevention, detection of pregnancy contractions, anticipation and monitoring of the ovulation period, effectiveness of some gynecology therapeutics, and supporting the discovery of new possible contraception methods.

In terms of future developments, we are considering the creation of a miniaturized intra-body biosensor that can be placed inside female cervix, as a hole, instead of placing only the thermistor. A new version of our application software for a Personal Digital Assistant (PDA) will be also considered.

\section{ACKNOWLEDGEMENT}

Part of this work has been supported by Instituto de Telecomunicações, Next Generation Networks and Applications Group (NetGNA), Portugal, and by the Euro-NF Network of Excellence of Seven Framework Programme of EU.

\section{REFERENCES}

[1] P. Kulkarni and Y. Öztürk, "Requirements and design spaces of mobile medical care," in ACM SIGMOBILE Mobile Computing and Communications Review. vol. 11, pp. 12 - 30, 2007.

[2] G. Shobha, R. R. Chittal, and K. Kumar, "Medical Applications of Wireless Networks," in Proceedings of the Second International Conference on Systems and Networks Communications: IEEE Computer Society, p. 82, 2007.

[3] A. Pantelopoulos and N. Bourbakis, "A survey on wearable biosensor systems for health monitoring," in Engineering in Medicine and Biology Society, 2008. EMBS 2008. 30th Annual International Conference of the IEEE, pp. 4887 - 4890, 2008.

[4] C. H. Chan, C. C. Y. Poon, R. C. S. Wong, and Y. T. Zhang, "A Hybrid Body Sensor Network for Continuous and Long-term Measurement of Arterial Blood Pressure," in International Summer School and Symposium on Medical Devices and Biosensors: 4th IEEE/EMBS, pp. 121 - 123, 2007.
[5] S. Patel, K. Lorincz, R. Hughes, N. Huggins, J. H. Growdon, M. Welsh, and P. Bonato, "Analysis of Feature Space for Monitoring Persons with Parkinson's Disease With Application to a Wireless Wearable Sensor System," in Engineering in Medicine and Biology Society, 2007. EMBS 2007. 29th Annual Int. Conference of the IEEE, pp. 6290-6293, 2007.

[6] W. D. Jones, "Taking Body Temperature, Inside Out," in IEEE Spectrum. vol. 43 Issue 1, pp. 13-15, 2006.

[7] U. Anliker, J. A. Ward, P. Lukowicz, G. Troster, F. Dolveck, M. Baer, F. Keita, E. B. Schenker, F. Catarsi, L. Coluccini, A. Belardinelli, D. Shklarski, M. Alon, E. Hirt, R. Schmid, and M. Vuskovic, " AMON: a wearable multiparameter medical monitoring and alert system," in IEEE Transactions on Information Technology in Biomedicine vol. 8, Issue 4, pp. 415 - 427, 2004.

[8] H. W. Taylor, S. E. Shidler, B. L. Lasley, L. Ngalamou, and F. E. Taylor, "FSH biosensor to detect postpartum ovarian recrudescence," in Engineering in Medicine and Biology Society. IEMBS '04. 26th Annual International Conference of the IEEE. vol. 1, pp. 1998 - 2001, 2004.

[9] F. Nebeker, "Golden accomplishments in biomedical engineering," in Engineering in Medicine and Biology Magazine, IEEE. vol. 21, pp. $17-47,2002$.

[10] J. Beaudoin and R. Marrocco, "Attentional validity effect across the human menstrual cycle varies with basal temperature changes," in Behavioural brain research. vol. 158, pp. 23-29, 2005.

[11] I. Campbella, "Body temperature and its regulation," in Anaesthesia \& Intensive Care Medicine. vol. 9, pp. 259-263, 2008.

[12] E. F. J. Ring, "Progress in the measurement of human body temperature," in Engineering in Medicine and Biology Magazine, IEEE. vol. 17, pp. 19-24, 1998

[13] L. Ngalamou and D. Rose, "Fertility information appliance," in Proceedings of the 15th IEEE Symposium on Computer-Based Medical Systems (CBMS 2002). pp. 335- 338, 2002.

[14] W. Jones, "Taking Body Temperature, Inside Out," IEEE Spectrum online, pp. 13-15, January 2006.

[15] DuoFertility, "http://www.duofertility.com", accessed in Feb. 2009.

[16] Z. McGreesh, N.E. Evans, and W.G. Scanlon, "Vaginal temperature sensing using UHF radio telemetry", Journal of Medical Engineering and Physics, Vol. 18, No. 2, pp. 110-114, March 1996.

[17] D.H Kosted, "A Method and System of Continual Temperature Monitoring", US Patent 20070027403, Feb 2007, http://www.freepatentsonline.com/US20070027403.html (accessed on December 2009). 